\title{
Investigating Other Syndrome in ESSENCE from a Data Quality Perspective
}

\author{
Dayaamayi Kurimella* and Jenna I. Johnson \\ Infectious Disease Epidemiology, LA Office of Public Health, New Orleans, LA, USA
}

\section{Objective}

This investigation takes a closer look at Other syndrome in ESSENCE and Null syndrome in LEEDS to understand what types of records are not tagged to a syndrome to elucidate data quality issues.

\section{Introduction}

The Louisiana Office of Public Health (OPH) Infectious Disease Epidemiology Section (IDEpi) conducts syndromic surveillance of Emergency Department (ED) visits through the Louisiana Early Event Detection System (LEEDS) and submits the collected data to ESSENCE. There are currently 86 syndromes defined in LEEDS including infectious disease, injury and environmental exposure syndromes, among others. LEEDS uses chief complaint, admit reason, and/or diagnosis fields to tag visits to relevant syndromes. Visits that do not have information in any of these fields, or do not fit any syndrome definition are tagged to Null syndrome. ESSENCE uses a different algorithm from LEEDS and only looks in chief complaint for symptom information to bin visits to syndromes defined in ESSENCE. Visits that do not fit the defined syndromes or do not contain any symptom information are tagged to Other syndrome. Since the transition from BioSense to ESSENCE, IDEpi has identified various data quality issues and has been working to address them. The NSSP team recently notified IDEpi that a large number of records are binning to Other syndrome, which led to the investigation of the possible underlying data quality issues captured in Other syndrome.

\section{Methods}

Daily submissions of electronic data are imported to and processed by LEEDS and ESSENCE for syndrome classification. LEEDS and ESSENCE were queried to first pull total visits and the percent of those visits tagged to Other syndrome in ESSENCE and Null syndrome in LEEDS between the dates of 1/01/2017 and 10/02/2017. The counts and percentages from both systems were compared. The percentage of total visits tagged to Other syndrome was stratified by facility to determine if there were significant differences between facilities. A line level review of visits tagged to Null syndrome in LEEDS and Other syndrome in ESSENCE was also conducted. This review showed that many records were pain related and many records were missing chief complaint. Both systems were then queried for the percent of visits in Other and Null syndrome that did not have symptom information and the percent of visits in Other and Null syndrome that mentioned "pain" in chief complaint.

\section{Results}

The average daily total visits in ESSENCE was 3279 visits per day compared to 5959 average visits per day in LEEDS, with counts in ESSENCE significantly dropping between 6/1/2017 and 7/1/2017. The average percentage of visits tagged to Other syndrome in ESSENCE was $63.16 \%$ while the percent of visits tagged to Null syndrome in LEEDS was $34.46 \%$. In ESSENCE, $24.22 \%$ of all visits tagged to other syndrome were pain related and $23.98 \%$ of all visits tagged to Other syndrome did not have any symptom information in chief complaint. In LEEDS, $43.03 \%$ of all visits tagged to Null syndrome were pain related and $3.6 \%$ of all visits tagged to Null syndrome had no symptom information. Finally, the percentage of total visits tagged to Other syndrome, stratified by facility, showed some facilities were disproportionately contributing to Other syndrome and that some facilities had major lapses in data in ESSENCE.

\section{Conclusions}

The dramatic difference in total visits between ESSENCE and LEEDS can be attributed to multiple reasons, most of which are likely related to the transition from BioSense to ESSENCE. This difference makes it difficult to compare data between the two systems, and IDEpi is continuing to work on understanding and resolving why these counts are so different. One of the reasons for the higher percentage of total visits binned to Other syndrome in ESSENCE compared to Null syndrome in LEEDS is related to the different processing methods of the two systems. LEEDS uses chief complaint, admit reason and diagnosis fields for symptom information, while ESSENCE only uses chief complaint. This allows LEEDS to tag more visits to syndromes other than Null syndrome. LEEDS also has more defined syndromes, which also contributes to the lower percentages of Null syndrome. The higher percentage of Other syndrome with no chief complaint in ESSENCE can partially be attributed to HL7 formatting issues. ESSENCE is not able to read chief complaint when it is populated if some HL7 formatting issues are present, while LEEDS is still able to read chief complaint when the same HL7 formatting issues exist. Finally, the percentage of total visits tagged to Other syndrome, stratified by facility has provided the facility level information necessary to address some of these data quality issues. Some of the facilities with lapses in data can be traced back to issues in the Master Facility Table (MFT), while other facilities have HL7 formatting issues that need to be addressed directly with the facility. In conclusion, exploring Other syndrome in ESSENCE can provide an interesting perspective into data quality. IDEpi's ability to compare Other syndrome in ESSENCE to Null syndrome in LEEDS has helped to further identify the data quality issues.

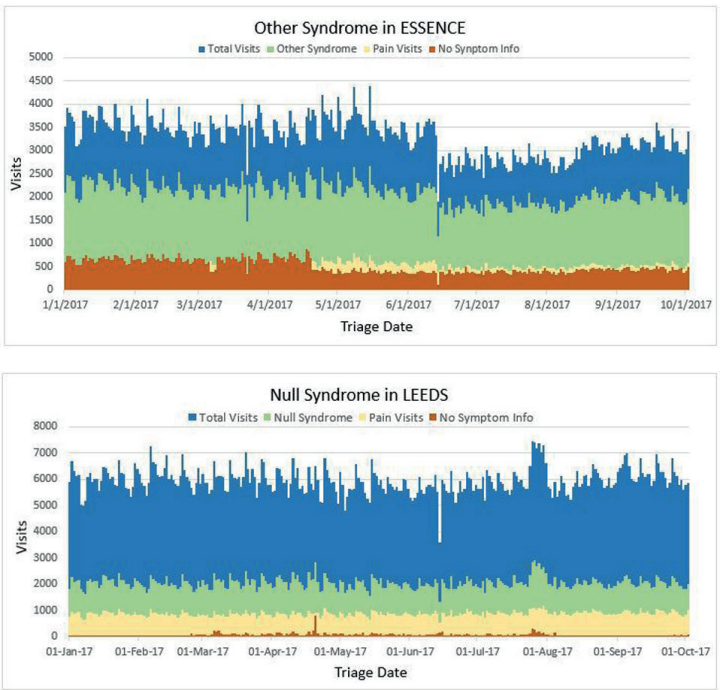




\section{ISDS 2018 Conference Abstracts}

Keywords

syndromic surveillance; Other syndrome; Data Quality

\section{Acknowledgments}

Michael Coletta, Aaron Kite Powell and Lakshmi Radhakrishnan

\section{*Dayaamayi Kurimella}

E-mail: Dayaamayi.Kurimella@la.gov 